\title{
Factores Pronósticos de Recurrencia y Mortalidad en los Pacientes con Tumor de Urotelio Superior Tratados con Cirugía
}

\section{Prognostic Factors for Recurrence and Mortality in Upper Urinary Tract Tumor}

\author{
Juan Aragón Chamizo ${ }^{1,2}$ Felipe Herranz Amo ${ }^{1}$ Carmen González Enguita ${ }^{1}$ \\ Carlos Hernández Fernández ${ }^{1}$ \\ ${ }^{1}$ Hospital General Universitario Gregorio Marañón, Madrid, Spain \\ 2 Hospital Universitario Fundación Jiménez Díaz, Madrid, Spain \\ Address for correspondence Juan Aragón Chamizo, MD, PhD, \\ Hospital General Universitario Gregorio Marañón, c/Antonio Arias, \\ 12, $5^{\circ} \mathrm{C}, 28009$, Madrid, Spain (e-mail: jaragonchamizo@gmail.com).
}

Urol Colomb 2018;27:55-62.
Resumen

\section{Palabras Claves}

- factores pronósticos

- tumor urotelial

- tumor de tracto urinario superior

- cirugía

- recurrencia

- mortalidad cáncerespecífica
Objetivo Identificar factores pronósticos de recurrencia y mortalidad cáncerespecífica en pacientes con tumor de urotelio superior tratados con cirugía.

Material y Métodos Análisis retrospectivo de pacientes con tumor de urotelio superior operados entre 1999 y 2011 (139 pacientes). Se recogieron variables demográficas, clínicas, diagnósticas y patológicas así como el tratamiento, las complicaciones y la evolución. Análisis descriptivo mediante la prueba de la Chi cuadrado para variables categóricas y el test ANOVA para variables continuas. Análisis univariante y multivariante mediante modelo de riesgos proporcionales de Cox. Significación estadística si $p<0,05$. Cálculos realizados con SPSS statistics v- 21 .

Resultados En el análisis multivariante se identificaron como factores predictores independientes de recurrencia: crecimiento sólido tumoral $(H R=4,02 ; p<0,001)$ y alto grado citológico (G3) ( $\mathrm{HR}=3,42 ; p=0,01)$. La presencia de tumor vesical previo o concomitante $(H R=1,84 ; p=0,07)$ presentó una tendencia a la significación. Se identificaron como factores predictores independientes de mortalidad cáncerespecífica: presencia de tumor vesical previo o concomitante $(H R=2,23 ; p=0,02)$, crecimiento sólido tumoral ( $H R=2,73 ; p=0,008)$, presencia de hidronefrosis $(H R=2,46 ; p=0,02)$ y estadio patológico avanzado pT3-pT4 $(H R=2,74 ; p=0,01)$. Conclusión En nuestra serie, la existencia de tumor vesical previo o sincrónico, el crecimiento tumoral sólido y el alto grado citológico se comportaron como factores pronósticos de recurrencia. La hidronefrosis, el tumor vesical previo o sincrónico, el estadio pT3-4 y el crecimiento tumoral sólido, se comportaron como factores pronósticos de mortalidad cáncer-específica. received October 10, 2016 accepted October 3, 2017 published online March 8, 2018
DOI https://doi.org/ $10.1055 / \mathrm{s}-0038-1637013$ ISSN 0120-789X. eISSN 2027-0119.
Copyright ( $)$ 2018, Sociedad Colombiana License terms de Urología. Publicado por Thieme Revinter Publicações Ltda., Rio de Janeiro, Brazil. Todos los derechos reservados.

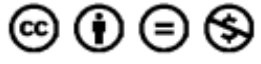




\section{Introducción}

El tumor de tracto urinario superior (TTUS), es una neoplasia infrecuente con una incidencia anual estimada en países desarrollados de 2 casos por cada 100000 habitantes. Es bien sabido que los dos factores de riesgo más importantes para el desarrollo de ese tumor son el tabaco y la exposición ocupacional. Sin embargo, el estudio de los factores que influyen en la recurrencia y la mortalidad es un tema que no cesa de actualizarse en los últimos años. El conocimiento de esos factores pronósticos puede ser por lo tanto de gran ayuda a la hora del manejo diagnóstico, terapéutico y durante el seguimiento. El objetivo de este trabajo es la identificación de los factores predictores de recurrencia y mortalidad cáncer-específica en los pacientes diagnosticados de TTUS y tratados con cirugía.

\section{Material y Método}

Análisis retrospectivo de 139 pacientes con TTUS tratados con cirugía entre 1999 y 2011 (12 años). La última actualización de los datos se realizó en julio de 2013. Se identificaron 144 pacientes con TTUS pero se excluyeron 4 pacientes que fueron tratados con vaporización láser a través de ureteroscopia y 1 que fue tratado con resección a través de nefrostomía percutánea debido a que el material obtenido era insuficiente para una correcta estadificación patológica, quedando por lo tanto 139 pacientes válidos para el estudio. De cada uno de los pacientes, se recogieron variables demográficas, clínicas, diagnósticas y patológicas así como el tratamiento realizado, las complicaciones acontecidas que se agruparon según la clasificación de Clavien-Dindo y la evolución.

La prueba de la Chi cuadrado se utilizó para evaluar la asociación entre variables categóricas y el test de ANOVA para evaluar las diferencias entre las variables con distribución continua.

Se realizó un análisis univariante y multivariante mediante un modelo de riesgos proporcionales de Cox (método enter). Para la depuración del modelo obtenido en el análisis multivariante se utilizó el método "atrás condicional" del programa SPSS, en el cual después de introducir todas las variables identificadas con significación estadística $(p<0,05)$ o con tendencia a la significación estadística $(p<0,1)$ en el análisis univariante, el programa en cada paso retira la variable con menor efecto sobre dicho modelo. En el análisis multivariante se habían incluido todas la variables que tenían una relación estadísticamente significativa con la recurrencia $(p<0,05)$ y las que presentaron una tendencia a la significación estadística $(p<0,1)$.

Todos los cálculos fueron realizados con el paquete estadístico IBM® SPSS $®$ statistics v-21. 


\section{Resultados}

\section{Características Clínicas, Patológicas y Quirúrgicas}

En la - Tabla 1 se puede observar una descripción detallada de las características clínicas. En la - Tabla 2 se detallan las características patológicas de la serie. En la - Tabla 3 se describen las variables relacionadas con la técnica quirúrgica, la vía de acceso y las complicaciones.

Tabla 1 Variables clínicas

\begin{tabular}{|c|c|}
\hline Variable & Valor \\
\hline Edad & $71 \pm 11,2(37-96)$ \\
\hline \multicolumn{2}{|l|}{ Sexo: } \\
\hline - Varón & $106(76,3 \%)$ \\
\hline - Mujer & $33(23,7 \%)$ \\
\hline \multicolumn{2}{|l|}{ Obesidad: } \\
\hline - Sí & $20(14,4 \%)$ \\
\hline - No & $119(85,6 \%)$ \\
\hline \multicolumn{2}{|l|}{ Índice de Charlson: } \\
\hline$\cdot 0$ & $37(26,6 \%)$ \\
\hline - 1 & $31(22,3 \%)$ \\
\hline - 2 & $30(21,6 \%)$ \\
\hline$\cdot \geq 3$ & $41(29,5 \%)$ \\
\hline \multicolumn{2}{|l|}{ Sintomatología: } \\
\hline - Hematuria & $80(57,6 \%)$ \\
\hline - Dolor lumbar & $18(12,9 \%)$ \\
\hline - S. Constitucional & $6(4,3 \%)$ \\
\hline \multicolumn{2}{|l|}{ Localización: } \\
\hline - Pelvis-cáliz & $77(55,4 \%)$ \\
\hline - Uréter & $51(36,7 \%)$ \\
\hline - Ambos & $11(7,9 \%)$ \\
\hline Hidronefrosis: & $78(56,1 \%)$ \\
\hline Tumor Vesical (antecedentes): & $42(30,2 \%)$ \\
\hline - Previo & $32(76,2 \%)$ \\
\hline - Sincrónico & $4(9,5 \%)$ \\
\hline - Ambos & $6(14,3 \%)$ \\
\hline \multicolumn{2}{|l|}{ - Grado Tumoral: } \\
\hline o G1 & $6(14,3 \%)$ \\
\hline o G2 & $17(40,5 \%)$ \\
\hline o G3 & $19(45,2 \%)$ \\
\hline \multicolumn{2}{|l|}{ - Infiltración: } \\
\hline o TVNMI & $37(88,1 \%)$ \\
\hline o TVMI & $5(11,9 \%)$ \\
\hline \multicolumn{2}{|l|}{ Citología urinaria: } \\
\hline - Negativa & $82(59 \%)$ \\
\hline - Sospechosa-positiva & $57(41 \%)$ \\
\hline
\end{tabular}

Tabla 1 (Continued)

\begin{tabular}{|l|l|}
\hline Variable & Valor \\
\hline Grado Tumoral: & \\
\hline$\bullet$ G1 & $1(0,7 \%)$ \\
\hline$\bullet$ G2 & $49(35,3 \%)$ \\
\hline$\bullet$ G3 & $89(64 \%)$ \\
\hline Estadio Local (cT): & \\
\hline$\bullet$ cTa & $17(12,2 \%)$ \\
\hline$\bullet$ cT1 & $71(51,1 \%)$ \\
\hline$\bullet$ cT2 & $16(11,5 \%)$ \\
\hline$\bullet$ cT3 & $29(20,9 \%)$ \\
\hline$\bullet$ cT4 & $6(4,3 \%)$ \\
\hline Estadio Ganglionar (cN): & \\
\hline$\bullet$ cN0 & $122(87,8 \%)$ \\
\hline$\bullet$ cN1 & $9(6,5 \%)$ \\
\hline$\bullet$ cN2 & $8(5,8 \%)$ \\
\hline Estadio a Distancia (cM): & \\
\hline$\bullet$ cM0 & $135(97,1 \%)$ \\
\hline$\bullet$ cM1 & $4(2,9 \%)$ \\
\hline
\end{tabular}

Tabla 2 Características patológicas

\begin{tabular}{|c|c|}
\hline \multicolumn{2}{|l|}{ Grado Patológico: } \\
\hline - G2 & $52(37,4 \%)$ \\
\hline - G3 & $87(62,6 \%)$ \\
\hline \multicolumn{2}{|l|}{ Estadio Patológico (pT): } \\
\hline - pTa & $18(12,9 \%)$ \\
\hline - pT1 & $62(44,6 \%)$ \\
\hline - $\mathrm{pT} 2$ & $21(15,1 \%)$ \\
\hline - pT3 & $32(23 \%)$ \\
\hline - pT4 & $6(4,3 \%)$ \\
\hline \multicolumn{2}{|l|}{ Afectación Linfática (pN): } \\
\hline - $\mathrm{pNx}$ & $118(84,9 \%)$ \\
\hline - $\mathrm{pNO}$ & $14(10,1 \%)$ \\
\hline - $\mathrm{pN} 1$ & $4(2,9 \%)$ \\
\hline - $\mathrm{pN2}$ & $2(1,4 \%)$ \\
\hline - $\mathrm{pN} 3$ & $1(0,7 \%)$ \\
\hline Tamaño tumoral (mm) & $3,8 \pm 2,5$ \\
\hline Ca “in situ” en la pieza & $16(11,5 \%)$ \\
\hline Multifocalidad & $24(17,3 \%)$ \\
\hline \multicolumn{2}{|l|}{ Tipo de Crecimiento: } \\
\hline - Papilar & $104(74,8 \%)$ \\
\hline - Sólido & $29(20,9 \%)$ \\
\hline - Ambos & $6(4,3 \%)$ \\
\hline Invasión linfovascular & $24(17,3 \%)$ \\
\hline Necrosis tumoral & $11(7,9 \%)$ \\
\hline Margen de resección afecto & $17(12,2 \%)$ \\
\hline
\end{tabular}


Tabla 3 Técnica quirúrgica, vías de acceso y complicaciones

\begin{tabular}{|c|c|}
\hline Variable & Valor \\
\hline \multicolumn{2}{|l|}{ Técnica Quirúrgica: } \\
\hline - Nefroureterectomía total & $109(78,4 \%)$ \\
\hline - Nefroureterectomía parcial & $18(12,9 \%)$ \\
\hline - Ureterectomía parcial & $12(8,6 \%)$ \\
\hline \multicolumn{2}{|l|}{ Vía de abordaje quirúrgico: } \\
\hline - Abierta & $55(39,6 \%)$ \\
\hline - Laparoscópica & $79(56,8 \%)$ \\
\hline - Laparoscópica reconvertida & $5(3,6 \%)$ \\
\hline \multicolumn{2}{|l|}{ Resección Rodete Vesical: } \\
\hline - Abierta & $35(25,2 \%)$ \\
\hline - Endoscópica & $48(34,5 \%)$ \\
\hline - Laparoscópica & $41(29,5 \%)$ \\
\hline - No extirpación & $15(10,8 \%)$ \\
\hline \multicolumn{2}{|l|}{ Linfadenectomía: } \\
\hline - Sí & $15(10,8 \%)$ \\
\hline - No & $124(89,2 \%)$ \\
\hline \multicolumn{2}{|l|}{ Complicaciones (Clavien-Dindo): } \\
\hline - $0-\mathrm{I}$ & $113(81,3 \%)$ \\
\hline$\cdot$ II & $17(12,2 \%)$ \\
\hline - Illa & $2(1,4 \%)$ \\
\hline - IIIb & $3(2,2 \%)$ \\
\hline - IVa & $2(1,4 \%)$ \\
\hline$\cdot \mathrm{V}$ & $2(1,4 \%)$ \\
\hline
\end{tabular}

\section{Factores Predictores de Recurrencia Tumoral}

Las variables relacionadas con la recurrencia (análisis univariante) se describen en la - Tabla 4.

En el análisis multivariante (-Tabla 4), el patrón de crecimiento sólido $(p<0,001)$ y el alto grado citológico (G3) $(p=0,004)$ se comportaron como factores predictores independientes de recurrencia tumoral. La existencia de tumor vesical previo o concomitante presentó una importante tendencia a la significación estadística $(p=0,076)$.

Factores Predictores de Mortalidad Cáncer-específica Las variables relacionadas con la mortalidad cáncerespecífica (análisis univariante), se detallan en la - Tabla 5.

En el análisis multivariante (-Tabla 5), se incluyeron todas la variables que tenían una relación estadísticamente significativa con la recurrencia $(p<0,05)$ y las que presentaron una tendencia a la significación $(p<0,1)$ (-Tabla 4). La existencia de hidronefrosis $(p=0,02)$, el tumor vesical previo o concomitante $(p=0,02)$ y el patrón de crecimiento sólido $(p=0,008)$ se comportaron como factores pronósticos independientes de mortalidad cáncer específica. El sexo femenino presentó una importante tendencia a la significación estadística $(p=0,06)$.

\section{Discusión}

Munoz y col. ${ }^{1}$, en una de las mayores series de TTUS publicadas con 9072 pacientes, observaron que con el paso del tiempo, la media de edad de los pacientes sube debido a un aumento en la esperanza de vida que da lugar a poblaciones más añosas. Ese aspecto coincide con lo encontrado en nuestra serie donde la mediana de edad es de 72 años.

La mayoría de las series publicadas sobre TTUS coinciden en afirmar que la edad se comporta como un factor predictivo de supervivencia ya que demuestran una menor supervivencia cáncer-específica y global en pacientes de edad avanzada. ${ }^{2}$ Esos hechos son atribuidos a la presencia de tumores biológicamente más agresivos en pacientes mayores. La edad avanzada es considerada por lo tanto, por esos autores, como un predictor de mayor agresividad del tumor. Sin embargo, la edad no debe ser en ningún caso un criterio de exclusión a la hora de valorar una cirugía radical ya que los pacientes añosos tratados mediante NUR presentan tasas inferiores de recurrencia tumoral. ${ }^{2}$

En nuestra serie, la edad guarda una relación con la recurrencia con una significación cercana a la significación estadística. Además, con respecto a la relación con la mortalidad cáncer-específica en el análisis univariante, la edad se comportó como un factor predictor de mortalidad cáncer-específica pero no en el análisis multivariante.

Con respecto a la distribución por sexo, encontramos una relación varón/mujer algo mayor en nuestra serie que en la mayoría de las series publicadas. Lehmann y col. describen un $67 \%$ de varones, ${ }^{3}$ Munoz y col. un $62 \%{ }^{1}$ y Jeldres y col. incluso un $59 \% .{ }^{4}$ La proporción de varones en nuestra serie es en cambio de un $76,3 \%$.

Cabe destacar en este sentido, un aumento en la incidencia de TTUS en mujeres en las últimas décadas. Dos grandes estudios multicéntricos no encontraron diferencias en las características patológicas entre varones y mujeres. 5,6 En cambio, se ha observado en algunas series que las mujeres pueden presentar tumores de más alto grado y estadio que los varones aunque una vez realizado el análisis multivariante, el sexo no afectaba a la supervivencia cáncer-específica. ${ }^{7}$ Según Fernández y col., esas diferencias podrían ser explicadas porque las mujeres tienen más edad en el momento de la cirugía que los varones. ${ }^{5}$

En nuestro estudio el sexo no guardó una relación estadísticamente significativa con la recurrencia de la enfermedad en los estudios multivariantes y se comportó como un factor predictivo de mortalidad cáncer-específica con tendencia a la significación estadística $(p=0,06)$. Sin embargo, la mayoría de estudios publicados insisten en que el sexo no debe ser considerado como factor predictivo de supervivencia. $^{7,8}$

El tabaco ha quedado establecido como un factor de riesgo para el desarrollo del tumor urotelial. Con respecto al pronóstico en el TTUS, se ha demostrado una mayor mortalidad cáncer-específica y una mayor recurrencia vesical en fumadores. ${ }^{9}$ Pese a que el mecanismo de carcinogénesis urotelial inducido por el tabaco no es bien 
Tabla 4 Factores predictores de recurrencia tumoral

\begin{tabular}{|c|c|c|c|c|c|c|c|c|c|}
\hline \multirow[b]{2}{*}{ Variable } & \multicolumn{3}{|c|}{ Análisis Univariante } & \multicolumn{3}{|c|}{ Análisis multivariante } & \multicolumn{3}{|c|}{ Método Atrás Condicional } \\
\hline & H.R. & IC95\% & $P$ & H.R. & IC95\% & $P$ & H.R. & IC95\% & $P$ \\
\hline Sexo: & & & 0,03 & & & 0,30 & & & \\
\hline - Varón & Ref. & & & Ref. & & & & & \\
\hline - Mujer & 2,03 & $1,03-3,98$ & & 1,58 & $0,65-3,83$ & & & & \\
\hline Afectación ganglionar (cN): & & & $<0,001$ & & & & & & \\
\hline - cNO & Ref. & & & & & & & & \\
\hline - cN1 & 4,33 & $2,08-9,04$ & & & & & & & \\
\hline Afectación metastásica (cM): & & & 0,06 & & & & & & \\
\hline - cM0 & Ref. & & & & & & & & \\
\hline - cM1 & 3,89 & $0,02-16,31$ & & & & & & & \\
\hline Tumor vesical(*): & & & 0,03 & & & 0,037 & & & 0,076 \\
\hline - No & Ref. & $1,03-$ & & Ref. & $1,05-$ & & Ref. & & \\
\hline - Sí & 1,98 & 3,80 & & 2,48 & 5,81 & & 1,87 & $0,93-3,75$ & \\
\hline Tipo de cirugía: & & & 0,03 & & & 0,068 & & & \\
\hline - Total & Ref. & & & Ref. & & & & & \\
\hline - Parcial & 2,48 & $1,07-5,74$ & & 8,39 & $0,85-82,37$ & & & & \\
\hline Tipo de acceso quirúrgico: & & & 0,07 & & & & & & \\
\hline - Laparoscópico & Ref. & & & & & & & & \\
\hline - Abierto & 1,82 & $0,95-3,48$ & & & & & & & \\
\hline Extirpación rodete vesical: & & & 0,02 & & & 0,68 & & & \\
\hline • Sí & Ref. & & & Ref. & & & & & \\
\hline - No & 2,61 & $1,14-5,97$ & & 1,11 & $0,25-3,36$ & & & & \\
\hline Estadio local (pT): & & & 0,001 & & & 0,30 & & & \\
\hline - pTa-1 & Ref. & & & Ref. & & & & & \\
\hline - $\mathrm{pT} 2$ & 1,52 & $0,58-3,97$ & 0,38 & 0,92 & $0,18-2,64$ & 0,58 & & & \\
\hline - $\mathrm{pT} 3-4$ & 3,88 & $1,92-7,84$ & $<0,001$ & 2,10 & $0,23-3,12$ & 0,80 & & & \\
\hline Estadio ganglionar (pN): & & & 0,01 & & & 0,73 & & & \\
\hline - pN0-x & Ref. & & & Ref. & & & & & \\
\hline$\cdot \mathrm{pN}+$ & 3,39 & $1,31-8,72$ & & 1,29 & $0,28-5,89$ & & & & \\
\hline Grado de diferenciación (G): & & & 0,003 & & & 0,06 & & & 0,014 \\
\hline$\cdot G 2$ & Ref. & & & Ref. & & & Ref. & & \\
\hline$\cdot \mathrm{G} 3$ & 3,51 & $1,54-7,99$ & & 3,37 & $0,92-12,28$ & & 2,83 & $1,24-6,67$ & \\
\hline Tipo de crecimiento tumoral: & & & $<0,001$ & & & 0,005 & & & $<0,001$ \\
\hline - Papilar & Ref. & & & Ref. & & & Ref. & & \\
\hline - Sólido & 4,09 & $2,16-7,76$ & & 3,86 & $1,49-10,01$ & & 3,42 & $1,28-9,14$ & \\
\hline Invasión linfovascular: & & & 0,001 & & & 0,68 & & & \\
\hline - No & Ref. & $1,53-6,07$ & & Ref. & $0,34-3,94$ & & & & \\
\hline - Sí & 3,05 & & & 0,76 & & & & & \\
\hline Márgenes quirúrgicos: & & & 0,04 & & & 0,78 & & & \\
\hline - Negativos & Ref. & & & Ref. & & & & & \\
\hline - Positivos & 2,31 & $1,01-5,29$ & & 0,81 & $0,18-3,66$ & & & & \\
\hline Quimioterapia adyuvante: & & & 0,008 & & & & & & \\
\hline - No & Ref. & & & & & & & & \\
\hline - Sí & 2,65 & $1,28-5,48$ & & & & & & & \\
\hline
\end{tabular}


Tabla 5 Factores Predictores de Mortalidad Cáncer Específica

\begin{tabular}{|c|c|c|c|c|c|c|c|c|c|}
\hline & Univa & iante & & Multi & ariante & & Atrás & Condicional & \\
\hline Variable & H.R. & IC95\% & $P$ & H.R. & IC95\% & $P$ & H.R. & IC95\% & $P$ \\
\hline Sexo: & & & 0,01 & & & 0,18 & & & 0,06 \\
\hline - Varón & Ref. & & & Ref. & & & Ref. & & \\
\hline - Mujer & 2,25 & $1,16-4,34$ & & 1,67 & $0,78-3,59$ & & 2,00 & $0,97-4,15$ & \\
\hline Edad & 1,03 & $1,00-1,07$ & 0,04 & 1,02 & $0,98-1,05$ & 0,23 & & & \\
\hline Hidronefrosis: & & & 0,06 & & & 0,023 & & & 0,02 \\
\hline - No & Ref. & & & Ref. & & & Ref. & & \\
\hline - Sí & 1,86 & $0,95-2,64$ & & 2,73 & $1,15-6,50$ & & 2,46 & $1,15-5,26$ & \\
\hline Tumor vesical( $\left.{ }^{*}\right)$ : & & & 0,07 & & & 0,048 & & & 0,02 \\
\hline - No & Ref. & & & Ref. & & & Ref. & & \\
\hline - Sí & 1,79 & $0,94-3,41$ & & 2,18 & $1,00-4,75$ & & 2,23 & $1,18-4,45$ & \\
\hline Tipo de NUR: & & & 0,04 & & & 0,20 & & & \\
\hline - Total & Ref. & & & Ref. & & & & & \\
\hline - Parcial & 2,37 & $1,03-5,46$ & & 2,02 & $0,67-6,08$ & & & & \\
\hline Tamaño tumoral: & 1,10 & $1,00-1,20$ & 0,048 & 1,07 & $0,95-1,21$ & 0,23 & & & \\
\hline Estadio local (pT): & & & 0,001 & & & 0,27 & & & 0,12 \\
\hline - pTa-1 & Ref. & & & Ref. & & & Ref. & & \\
\hline - $\mathrm{pT} 2$ & 1,54 & $0,59-3,98$ & 0,36 & 0,67 & $0,19-2,36$ & 0,54 & 0,64 & $0,18-2,18$ & 0,47 \\
\hline - pT3-4 & 3,58 & $1,80-7,15$ & $<0,001$ & 1,78 & $0,65-4,86$ & 0,25 & 2,74 & $1,22-6,15$ & 0,014 \\
\hline Estadio ganglionar $(\mathrm{pN})$ : & & & 0,03 & & & 0,54 & & & \\
\hline - $\mathrm{pNO} 0-\mathrm{x}$ & Ref. & & & Ref. & & & & & \\
\hline - $\mathrm{pN}+$ & 2,72 & $1,06-6,96$ & & 1,59 & $0,34-7,23$ & & & & \\
\hline Grado de diferenciación (G): & & & 0,009 & & & 0,43 & & & \\
\hline$\cdot \mathrm{G} 2$ & Ref. & $1,28-5,74$ & & Ref. & & & & & \\
\hline$\cdot \mathrm{G} 3$ & 2,71 & & & 1,44 & $0,58-3,57$ & & & & \\
\hline Tipo de crecimiento tumoral: & & & 0,001 & & & 0,15 & & & 0,008 \\
\hline - Papilar & Ref. & & & Ref. & & & Ref. & & \\
\hline - Sólido & 3,04 & $1,61-5,76$ & & 1,95 & $0,78-4,90$ & & 2,73 & $1,29-5,78$ & \\
\hline Invasión linfovascular: & & & 0,002 & & & 0,13 & & & \\
\hline - No & Ref. & & & Ref. & & & & & \\
\hline - Sí & 2,92 & $1,47-5,78$ & & 1,99 & $0,80-4,971$ & & & & \\
\hline
\end{tabular}

conocido, parece evidente que la escalada de dosis y de duración en el consumo de tabaco aumenta el riesgo de desarrollo y progresión de la enfermedad. Rink y col., encuentran una relación proporcional entre la cantidad de tabaco consumido y el riesgo de desarrollo de enfermedad avanzada de manera que los grandes fumadores de larga evolución de su serie tienen más riesgo de presentar tumores especialmente agresivos (estadios avanzados, afectación ganglionar...) así como peores cifras de recurrencia y de mortalidad cáncer-específica. ${ }^{10}$ Ese grupo también objetiva que el cese de consumo de tabaco durante más de 10 años parece mitigar los efectos desfavorables del tabaco en pacientes con TTUS. ${ }^{10}$ En nuestra serie, no ha sido posible evaluar el efecto del tabaquismo debido a la falta de datos en las historias clínicas.
Ha quedado establecido en la literatura que la presencia de síntomas sistémicos (pérdida de peso, anorexia, dolor óseo...), se relaciona tanto con TTUS de mayor grado y estadio ${ }^{11}$ como con peores cifras de supervivencia global. ${ }^{12}$ En cambio, con respecto a la sintomatología local (dolor local, hematuria), no se han encontrado diferencias en la supervivencia al comparar a pacientes sintomáticos frente a pacientes asintomáticos.

En nuestra serie, ningún tipo de síntoma, ni local ni sistémico, guardaba relación con la recidiva tumoral ni con la mortalidad cáncer-específica, hecho seguramente debido a que solo incluimos pacientes tratados con cirugía y excluimos pacientes con afectación metastática extensa que son los que tienen más probabilidad de tener sintomatología sistémica. 
La localización tumoral es una de las variables que más controversia genera, hasta el punto de que es uno de los grandes cambios observados en las guías europeas en los últimos años: mientras que en el año 2011 se aseveraba que la localización tumoral no era un factor pronóstico, en la actualización de 2014 se afirma que la localización del tumor tiene importancia pronóstica. Pese a eso, en publicaciones recientes, algunos autores sugieren utilizar la multifocalidad más que la localización tumoral como factor de riesgo de recurrencia, de igual modo que ocurre en el cáncer vesical. ${ }^{13}$

Varios son los estudios multicéntricos que concluyen que la localización tumoral no es un factor pronóstico independiente ya que encuentran cifras similares de supervivencia libre de recurrencia y de supervivencia cáncer-específica en pacientes con tumores ureterales y en pacientes con tumores pielocaliciales. ${ }^{14-16}$ Nuestros datos están a tono con esos estudios.

En cambio otras publicaciones demuestran que la localización tumoral se comporta como un factor predictor independiente de supervivencia, de manera que los tumores ureterales presentan un peor pronóstico que los pielocaliciales. ${ }^{17}$ El trabajo con más peso en ese sentido, es el estudio francés multicéntrico que defiende que los pacientes con TTUS ureterales tienen peor pronóstico en cuanto a la supervivencia. ${ }^{18}$ Park y col., sugieren un papel protector del parénquima renal gracias al cual los tumores pT3 pielocaliciales, serían menos agresivos que los pT3 ureterales. ${ }^{19}$

La hidronefrosis es considerada por varios autores como factor pronóstico hasta el punto de que no solo su presencia conlleva consecuencias pronósticas sino que también el grado de hidronefrosis estaría relacionado con la supervivencia. $^{20}$ Además se ha demostrado la relación entre la presencia de hidronefrosis y la de un estadio tumoral avanzado dando a entender que la patogénesis de dicha hidronefrosis no sería solamente la obstrucción de la vía urinaria. ${ }^{21} \mathrm{Ng}$ y col., han demostrado que la presencia de hidronefrosis preoperatoria, se relaciona con el desarrollo de metástasis y con la supervivencia cáncer-específica. ${ }^{22}$ En nuestra serie, la presencia de hidronefrosis no se relacionó con la recidiva tumoral pero sí se comportó como un factor predictor independiente de mortalidad cáncer-específica, como concluye $\mathrm{Ng}$ en su estudio. ${ }^{23}$

Basándose en la teoría de que el TTUS forma parte de una enfermedad "panurotelial" que afecta a todo el tracto urinario recubierto por urotelio (desde los cálices renales hasta la uretra distal), algunos autores defienden que su pronóstico puede verse afectado por la presencia (sincrónica o metacrónica) de un tumor vesical. Mullerad y col., objetivan que el antecedente de tumor vesical previo o sincrónico en un paciente con TTUS, se relaciona con tasas menores de supervivencia libre de recidiva y de supervivencia cáncerespecífica. $^{24}$ Del mismo modo, cifras inferiores de supervivencia cáncer-específica se relacionan también con la presencia de tumor vesical músculo-infiltrante en el momento de la NUR. ${ }^{23}$

De manera similar a la descrita en la literatura, en nuestra serie, la presencia de un tumor vesical en el momento de la
NUR se comportó como un factor predictor de recidiva. Así la supervivencia libre de recidiva a los 5 años en los enfermos sin antecedente de tumor vesical fue de un $76,3 \%$ frente a un 56,3\% para los pacientes con tumor vesical previo o sincrónico. Al fijarnos en la supervivencia, la presencia de tumor vesical previo o simultáneo al diagnóstico de TTUS, se comportó como factor predictor independiente de mortalidad cáncer-específica, compartiendo así ese resultado con lo descrito en la literatura. ${ }^{23}$

Mención especial debido a la importancia actual del tema merece la afectación linfática que siempre ha sido considerada como un factor pronóstico del TTUS puesto que traduce la rápida diseminación de las células tumorales a las áreas linfáticas regionales. Varios autores han publicado en la literatura cómo empeora la supervivencia en pacientes con afectación linfática ${ }^{25,26}$ : la supervivencia cáncer-específica en casos de metástasis linfáticas no pasa del $30 \%$. Además, está descrito que la extensión extranodal es un factor predictor importante en pacientes con TTUS $\mathrm{N}+{ }^{27}$

En nuestro estudio no hemos podido analizar si la raza se comporta como un factor predictivo de recurrencia ya que la totalidad de los pacientes estudiados en nuestra serie son de raza caucásica. Actualmente, lo descrito en la literatura sobre ese aspecto, es un aumento de la incidencia del TTUS en todos los grupos raciales y una menor supervivencia en los pacientes de raza negra al compararlos con el resto de las razas. $^{28}$

Otra variable que parece tener relación con el TTUS aunque en nuestro estudio no se identificó como factor predictor, es la obesidad. Ehdaie y col., objetivan que la obesidad, considerando como tal un IMC superior a 30, se relaciona con peores cifras de supervivencia libre de recidiva, supervivencia cáncer-específica y supervivencia global. ${ }^{29}$ La explicación a esa observación se basa en que los pacientes obesos tienen con frecuencia tumores con características más agresivas. Además el exceso de grasa corporal se asocia a su vez con mayor inflamación sistémica y niveles elevados de factor de crecimiento insulin-like, estrechamente emparentados con fenómenos de proliferación celular y apoptosis.

La limitación principal de nuestro trabajo, es que se trata de un estudio retrospectivo; además, al tratarse de pacientes de un solo centro, el número de pacientes es significativamente inferior al de los estudios publicados en la literatura. Nuestro objetivo es aumentar la cifra de pacientes de nuestra serie con el paso del tiempo para obtener así resultados de mayor consistencia. Nos planteamos también en un futuro, y con el mismo objetivo, la posibilidad de participar en estudios multicéntricos similares a los que aparecen en la literatura.

\section{Conclusión}

En nuestra serie, la existencia de tumor vesical previo o sincrónico, el crecimiento tumoral sólido y el alto grado citológico, se comportaron como factores pronósticos de recurrencia. La hidronefrosis, el tumor vesical previo o sincrónico, el estadio pT3-4 y el crecimiento tumoral sólido, se comportaron como factores pronósticos de mortalidad cáncer-específica. 


\section{Responsabilidades Éticas}

Protección de personas y animales. Los autores declaran que para esta investigación no se han realizado experimentos en seres humanos ni en animales.

Confidencialidad de los datos. Los autores declaran que han seguido los protocolos de su centro de trabajo sobre la publicación de datos de pacientes.

Derecho a la privacidad y consentimiento informado. Los autores declaran que en este artículo no aparecen datos de pacientes.

\section{Conflictos de Interés}

No existen conflictos de interés por parte de los autores.

\section{Referencias}

1 Munoz JJ, Ellison LM. Upper tract urothelial neoplasms: incidence and survival during the last 2 decades. J Urol 2000;164(05): 1523-1525

2 Chromecki TF, Ehdaie B, Novara G, et al. Chronological age is not an independent predictor of clinical outcomes after radical nephroureterectomy. World J Urol 2011;29(04):473-480

3 Lehmann J, Suttmann H, Kovac I, et al. Transitional cell carcinoma of the ureter: prognostic factors influencing progression and survival. Eur Urol 2007;51(05):1281-1288

4 Jeldres C, Sun M, Isbarn $\mathrm{H}$, et al. A population-based assessment of perioperative mortality after nephroureterectomy for uppertract urothelial carcinoma. Urology 2010;75(02):315-320

5 Fernández MI, Shariat SF, Margulis V, et al. Evidence-based sexrelated outcomes after radical nephroureterectomy for upper tract urothelial carcinoma: results of large multicenter study. Urology 2009;73(01):142-146

6 Shariat SF, Favaretto RL, Gupta A, et al. Gender differences in radical nephroureterectomy for upper tract urothelial carcinoma. World J Urol 2011;29(04):481-486

7 Cosentino M, Palou J, Gaya JM, Breda A, Rodriguez-Faba O, Villavicencio-Mavrich $\mathrm{H}$. Upper urinary tract urothelial cell carcinoma: location as a predictive factor for concomitant bladder carcinoma. World J Urol 2013;31(01):141-145

8 Lughezzani G, Burger M, Margulis V, et al. Prognostic factors in upper urinary tract urothelial carcinomas: a comprehensive review of the current literature. Eur Urol 2012;62(01):100-114

9 Simsir A, Sarsik B, Cureklibatir I, Sen S, Gunaydin G, Cal C. Prognostic factors for upper urinary tract urothelial carcinomas: stage, grade, and smoking status. Int Urol Nephrol 2011;43(04):1039-1045

10 Rink M, Xylinas E, Margulis V, et al; Upper Tract Urothelial Carcinoma Collaboration. Impact of smoking on oncologic outcomes of upper tract urothelial carcinoma after radical nephroureterectomy. Eur Urol 2013;63(06):1082-1090

11 Raman JD, Shariat SF, Karakiewicz PI, et al; Upper-Tract Urothelial Carcinoma Collaborative Group. Does preoperative symptom classification impact prognosis in patients with clinically localized upper-tract urothelial carcinoma managed by radical nephroureterectomy? Urol Oncol 2011;29(06):716-723

12 Inman BA, Tran VT, Fradet Y, Lacombe L. Carcinoma of the upper urinary tract: predictors of survival and competing causes of mortality. Cancer 2009;115(13):2853-2862
13 Williams AK, Kassouf W, Chin J, et al. Multifocality rather than tumor location is a prognostic factor in upper tract urothelial carcinoma. Urol Oncol 2013;31(07):1161-1165

14 Isbarn H, Jeldres C, Shariat SF, et al. Location of the primary tumor is not an independent predictor of cancer specific mortality in patients with upper urinary tract urothelial carcinoma. J Urol 2009;182(05):2177-2181

15 Favaretto RL, Shariat SF, Chade DC, et al. The effect of tumor location on prognosis in patients treated with radical nephroureterectomy at Memorial Sloan-Kettering Cancer Center. Eur Urol 2010;58(04):574-580

16 Raman JD, Ng CK, Scherr DS, et al. Impact of tumor location on prognosis for patients with upper tract urothelial carcinoma managed by radical nephroureterectomy. Eur Urol 2010;57(06): 1072-1079

17 Akdogan B, Dogan HS, Eskicorapci SY, Sahin A, Erkan I, Ozen H. Prognostic significance of bladder tumor history and tumor location in upper tract transitional cell carcinoma. J Urol 2006; 176(01):48-52

18 Ouzzane A, Colin P, Xylinas E, et al; French Collaborative National Database on UUT-UC. Ureteral and multifocal tumours have worse prognosis than renal pelvic tumours in urothelial carcinoma of the upper urinary tract treated by nephroureterectomy. Eur Urol 2011;60(06):1258-1265

19 Park J, Ha SH, Min GE, et al. The protective role of renal parenchyma as a barrier to local tumor spread of upper tract transitional cell carcinoma and its impact on patient survival. J Urol 2009;182(03):894-899

20 Cho KS, Hong SJ, Cho NH, Choi YD. Grade of hydronephrosis and tumor diameter as preoperative prognostic factors in ureteral transitional cell carcinoma. Urology 2007;70(04):662-666

21 Ito Y, Kikuchi E, Tanaka N, et al. Preoperative hydronephrosis grade independently predicts worse pathological outcomes in patients undergoing nephroureterectomy for upper tract urothelial carcinoma. J Urol 2011;185(05):1621-1626

$22 \mathrm{Ng} \mathrm{CK}$, Shariat SF, Lucas SM, et al. Does the presence of hydronephrosis on preoperative axial CT imaging predict worse outcomes for patients undergoing nephroureterectomy for upper-tract urothelial carcinoma? Urol Oncol 2011;29(01):27-32

23 Novara G, De Marco V, Gottardo F, et al. Independent predictors of cancer-specific survival in transitional cell carcinoma of the upper urinary tract: multi-institutional dataset from 3 European centers. Cancer 2007;110(08):1715-1722

24 Mullerad M, Russo P, Golijanin D, et al. Bladder cancer as a prognostic factor for upper tract transitional cell carcinoma. J Urol 2004;172(6 Pt 1):2177-2181

25 Margulis V, Shariat SF, Matin SF, et al; Upper Tract Urothelial Carcinoma CollaborationThe Upper Tract Urothelial Carcinoma Collaboration. Outcomes of radical nephroureterectomy: a series from the Upper Tract Urothelial Carcinoma Collaboration. Cancer 2009;115(06):1224-1233

26 Lughezzani G, Jeldres C, Isbarn $\mathrm{H}$, et al. A critical appraisal of the value of lymph node dissection at nephroureterectomy for upper tract urothelial carcinoma. Urology 2010;75(01):118-124

27 Fajkovic H, Cha EK, Jeldres C, et al. Prognostic value of extranodal extension and other lymph node parameters in patients with upper tract urothelial carcinoma. J Urol 2012;187(03):845-851

28 Raman JD, Messer J, Sielatycki JA, Hollenbeak CS. Incidence and survival of patients with carcinoma of the ureter and renal pelvis in the USA, 1973-2005. BJU Int 2011;107(07):1059-1064

29 Ehdaie B, Chromecki TF, Lee RK, et al. Obesity adversely impacts disease specific outcomes in patients with upper tract urothelial carcinoma. J Urol 2011;186(01):66-72 